\title{
A influência das orientações motivacionais nas atitudes despor- tivas em aulas de Educação Física
}

\author{
Universidade de Trás-os-Montes e Alto Douro \\ ${ }^{2}$ Escola EB 2,3/S Abel Botelho (Tabuaço) \\ ${ }^{3}$ Escola EB 2,3/S Dr. José Casimiro Matias (Almeida) \\ Fernandes, H.; Vasconcelos-Raposo, J.; Moreira, M.; \\ Costa, H.; A influência das orientações motiva- \\ cionais nas atitudes desportivas em aulas de \\ Educação Física. Motricidade 3(3): 16-23
}

Hélder Fernandes ${ }^{1}$, José Vasconcelos-Raposo', Miguel Moreira ${ }^{2}$, Henrique Costa ${ }^{3}$

${ }^{1}$ Centro de Estudos em Desenvolvimento Humano, Actividade Física e Saúde

\section{Resumo}

O presente estudo pretendeu examinar os valores desportivos e orientações motivacionais expressos por alunos de Educação Física, perante diferentes situações desportivas e comparar os comportamentos desses mesmos, de acordo com as variáveis sexo, faixa etária e envolvimento desportivo. A amostra deste estudo foi constituída por 422 alunos (221 do sexo masculino e 201 do sexo feminino), com uma média de idades de 13.73 \pm 2.46 anos, tendo idades compreendidas entre os 9 e os 21 anos de idade. Os instrumentos utilizados foram o Questionário de Valores no Desporto e o Questionário de Orientação Motivacional no Desporto ${ }^{5}$. Numa análise generalista, os indivíduos demonstraram concordar em assumir atitudes perante situações desportivas, consoante os princípios básicos do desportivismo e fair-play. A variável que mais contribuiu para uma diferenciação significativa foi a faixa etária, evidenciando os alunos com idades compreendidas entre os 13 e 15 anos, como menos concordantes com atitudes desportivas e revelando uma orientação para o ego. Como tal, propomos que os agentes de socialização presentes no contexto desportivo, devem a seu nível respectivo e em função das suas possibilidades de intervenção, zelar pelo respeito deste princípio ${ }^{4}$.

Palavras-chave: valores desportivos, orientações motivacionais e Educação Física.

\section{Abstract}

Influence of motivacional orientation in sports behaviour / attitude in Physical Education classes

The purposes of the present study were twofold: (i) to examine the levels of sportsmanship and goal orientations in Physical Education students and (ii) to compare those same levels according to different sexes, ages and sport's involvement. A sample of 422 students (221 male and 201 female) with a mean age of $13.73 \pm 2.46$ years (ages comprised between 9 and 21 years old), completed two questionnaires: a) the Questionnaire of Sport's Values ${ }^{7}$ and b) a Portuguese version of the Task and Ego Orientation in Sport Questionnaire 5 . In general, the students stated to display behaviors in sport's settings, according to the basic principles of sportsmanship and fair play. The independent variable that most contributed to a significant demarcation in Physical Education was the student's age, whilst those with ages between 13 and 15 years stating to not display sportsmanship behaviours and to posses high levels of ego orientation. Overall, we recommend that the sport's socialization agents should endorse the sportsmanship's principles deference, at their respective levels and in function of their intervention possibilities ${ }^{4}$.

Keywords: sportsmanship, goal orientations and fair-play. 


\section{Introdução}

Tendo em conta o envolvimento desportivo que cada vez mais é notório nas camadas jovens, já foram identificadas algumas consequências positivas dessa participação social. No entanto, vários investigadores na área da Psicologia do Desporto, e em especial no processo de socialização dos jovens através da prática desportiva, assinalam que esses resultados se apresentam contraditórios ${ }^{6}$.

Alguns comportamentos que têm vindo a ser mais frequentes são: fazer batota, praticar agressões, adoptar comportamentos violentos e faltar ao respeito a adversários e árbitros. Embora as ocorrências mais frequentes e visíveis sejam observáveis no desporto profissional, já se começa a encontrar cada vez mais esses maus comportamentos no desporto jovem ${ }^{1}$.

Todavia, a implementação e desenvolvimento de programas desportivos para jovens, são habitualmente baseados no pressuposto, que a prática desportiva é um factor importante na educação dos praticantes. No entanto, a educação dos valores não é ainda considerado como fundamental na disciplina de Educação Física?. Tal facto ainda se agrava, quando se verifica que os comportamentos inapropriados são cada vez mais frequentes nas aulas de Educação Física, justificando por isso uma análise atenta da sua ocorrência e natureza, de forma a caracterizar esta situação ${ }^{11}$.

Deste modo, os objectivos do presente estudo foram: (i) examinar os valores desportivos e orientações cognitivas, expressos por alunos de Educação Física, perante diferentes situações desportivas e, (ii) comparar os comportamentos desses mesmos, de acordo com diferentes situações de envolvimento desportivo.

\section{Metodologia}

\section{Amostra}

A amostra deste estudo foi constituída por 422 alunos (221 do sexo masculino e 201 do sexo feminino), com uma média de idades de 13.73 \pm \pm 2.46 anos, tendo idades compreendidas entre os 9 e os 21 anos de idade (amostragem não probabilística de conveniência). As faixas etárias foram delimitadas considerando a proposta de Weiss 16: < 12 anos (dos 9 aos 12 anos), 13-15 anos (dos 13 aos 15 anos) e $>16$ anos (dos 16 aos 21 anos).

\section{Procedimentos}

Os instrumentos utilizados foram o Questionário de Valores no Desporto $(\mathrm{QVD})^{7}$ e o Questionário de Orientação Motivacional no Desporto (QOMD) adaptado para a língua portuguesa por Fonseca e Biddle ${ }^{5}$. O primeiro consta de 12 itens e o segundo é constituído por 13 itens, pelo que ambos são respondidos de acordo com uma escala tipo Likert de 5 pontos (1: discordo totalmente a 5: concordo totalmente).

\section{Estatística}

Inicialmente, efectuou-se uma Análise Factorial de Componentes Principais (AFCP), com rotação Varimax e respectiva análise de consistência interna ( $\alpha$ de Cronbach), para cada um dos factores obtidos. É de salientar que para a obtenção da solução factorial final dos diferentes instrumentos, os critérios utilizados para reter um item num factor, foram os seguintes: (i) saturação igual ou superior a 0.40 no hipotético factor; (ii) o item não devia ser redundante com outro item no mesmo factor; e, (iii) a retirada do item não devia fazer baixar a fidelidade do suposto factor.

Após a obtenção da estrutura factorial de cada instrumento, procedeu-se à análise comparativa das variáveis independentes (sexo, faixa etária e envolvimento desportivo), através do teste $t$ de Student para amostras independentes e da análise de variância (ANOVA) one-way com respectivo teste post-hoc Schéffe. Para análise da relação preditiva entre variáveis, recorreu-se ao modelo de regressão linear simples. O nível de significância foi mantido em 5\% ( $p<0.05)$. 


\section{Resultados}

No presente capítulo são expostos os resultados obtidos a partir dos dados recolhidos, pelo que por uma questão de simplicidade, unicamente apresentamos os resultados onde se verificaram diferenças estatisticamente significativas nas dimensões psicológicas que são objecto de estudo na presente investigação. Todavia, esses mesmos dados/resultados estão disponíveis a pedido ao primeiro autor.
A partir da análise factorial obtiveram-se dois factores para cada questionário, sendo: QVD ("Atitudes anti-desportivas" $-31.9 \%$ de variância e $\alpha$ de Cronbach de 0.83; "Atitudes desportivas" - 27.0\% de variância e $\alpha$ de Cronbach de 0.72) e QOMD ("Orientação para a tarefa/mestria" $-25.2 \%$ de variância e $\alpha$ de Cronbach de 0.79; (“Orientação para o ego" - 23.1\% de variância e $\alpha$ de Cronbach de 0.81).

No quadro 1 é apresentada a estrutura factorial do QVD.

Quadro 1: Estrutura factorial do Questionário de Valores no Desporto.

$\begin{array}{lcc}\text { Item } & \text { Factor } 1 & \text { Factor } 2 \\ \text { Atitudes } \\ \text { Desportivas }\end{array}$

6. Quem joga sempre de forma correcta, respeitando os adversários, os árbitros ou os juízes, perde quase sempre.

0,685

11. Se o árbitro se engana a nosso favor, aceito a sua decisão, mas se engana contra mim ou contra a minha equipa, devo discordar e protestar fortemente.

7. É preferível ganhar mesmo fazendo batota, do que perder sendo correcto, respeitando os adversários, árbitros ou juízes.

4. Acho correcto fazer "batota", desde que o árbitro não veja.

12. Num jogo ou numa competição que já não posso ganhar ou fazer uma boa marca, deixo de me esforçar.

8. Quando jogo mal ou faço um mau resultado, arranjo desculpas para me justificar.

3. No final de uma competição, quer ganhe ou perca, cumprimento os adversários.

5. Prefiro jogar (competir) e perder, do que ganhar por falta de comparência do adversário.

2. Apoio e ajudo todos os meus colegas de equipa, mesmo quando eles são "mais fracos" do que eu, ou quando cometem erros.

10. Ganhar é somente uma parte importante (mas não a mais importante) do prazer de jogar (competir). 
De acordo com a análise estatística efectuada, o item 9 "...quando o árbitro ou o juiz tem dúvidas sobre quem foi o infractor ou o responsável pela violação de uma regra, sou capaz de me denunciar en próprio como responsável.", não respeitou os critérios definidos para a saturação do item no factor, pelo que quando adicionado a qualquer um dos factores, fez baixar o valor de consistência interna. Como tal, foi eliminado da análise estatística realizada.

No quadro 2 são apresentadas as saturações factoriais dos itens do QOMD, nas duas dimensões obtidas a partir da AFCP.

Quadro 2: Estrutura factorial do Questionário de Orientação Motivacional no Desporto.

\begin{tabular}{lcc} 
Item & $\begin{array}{c}\text { Factor 1 } \\
\text { Orientação } \\
\text { Tarefa }\end{array}$ & $\begin{array}{c}\text { Factor } 2 \\
\text { Orientação } \\
\text { Ego }\end{array}$ \\
\hline 10. Algo que aprendo me faz querer continuar e praticar mais. & 0.738 & \\
7. Aprendo uma nova técnica esforçando-me bastante. & 0.699 & \\
2. Aprendo uma nova técnica e isso me faz querer praticar mais. & 0.695 & \\
8. Trabalho realmente bastante. & 0.686 & 0.750 \\
5. Aprendo algo que dá prazer em fazer. & 0.648 & 0.739 \\
12. Sinto que uma técnica que aprendo está bem. & 0.633 & 0.735 \\
13. Faço o meu melhor. & 0.497 & 0.697 \\
11. Sou o melhor. & & 0.669 \\
6. Os outros cometem erros e eu não. & & 0.662 \\
3. Consigo fazer melhor do que os meus amigos. & & \\
1. Sou o único que consegue executar as técnicas. & & \\
4. Os outros não conseguem fazer tão bem como eu. & & \\
9. Ganho a maioria das provas. & & \\
\hline
\end{tabular}

Numa perspectiva de análise generalista, os alunos demonstraram concordar em assumir atitudes perante situações desportivas, consoante os princípios básicos do desportivismo e fair-play. Contudo, quando comparando os dados de acordo com as diferentes variáveis independentes, e verificando a influência da orientação para tarefa nas atitudes desportivas, constata-se o seguinte apresentado no quadro 3.
No geral, verifica-se que os elementos femininos concordam menos do que os elementos masculinos, em demonstrar atitudes anti-desportivas, revelando assim uma menor orientação para o ego.

No quadro 4 são apresentados os resultados da análise comparativa por faixas etárias, no que concerne as diferentes subescalas do QVD e QOMD. 
Quadro 3: Análise comparativa por sexo.

\begin{tabular}{lcccc} 
Factor & Rapazes & Raparigas & $\mathbf{t}$ & $\mathbf{p}$ \\
\hline Atitudes Anti-desportistas & $2.26 \pm 0.81$ & $1.90 \pm 0.77$ & 4.716 & $\mathbf{0 . 0 0 0}$ \\
Orientação para o Ego & $2.52 \pm 0.98$ & $2.26 \pm 0.91$ & 2.779 & $\mathbf{0 . 0 0 6}$ \\
\hline
\end{tabular}

Quadro 4: Análise comparativa por faixa etária.

\begin{tabular}{lccccc} 
Factor & $<\mathbf{1 2}$ anos & $\mathbf{1 3}$ a $\mathbf{1 6}$ anos & $>\mathbf{1 6}$ anos & $\mathbf{t}$ & $\mathbf{p}$ \\
\hline Atitudes Desportistas & $3.83 \pm 0.74$ & $3.68 \pm 0.66$ & $3.93 \pm 0.48$ & 5.371 & $\mathbf{0 . 0 0 5}$ \\
Atitudes Anti-desportistas & $2.09 \pm 0.93$ & $2.19 \pm 0.77$ & $1.91 \pm 0.69$ & 4.050 & $\mathbf{0 . 0 1 8}$ \\
Orientação para a Tarefa & $4.26 \pm 0.68$ & $4.01 \pm 0.71$ & $4.18 \pm 0.67$ & 5.158 & $\mathbf{0 . 0 0 6}$ \\
Orientação para o Ego & $2.59 \pm 1.03$ & $2.40 \pm 0.96$ & $2.10 \pm 0.75$ & 7.929 & $\mathbf{0 . 0 0 0}$ \\
\hline
\end{tabular}

Quadro 5: Análise comparativa por envolvimento desportivo.

\begin{tabular}{lccccc} 
Factor & $\begin{array}{c}\text { Não } \\
\text { praticantes }\end{array}$ & $\begin{array}{c}\text { Modalidade } \\
\text { Colectiva }\end{array}$ & $\begin{array}{c}\text { Modalidade } \\
\text { Individual }\end{array}$ & F & p \\
\hline Orientação para a Tarefa & $4.03 \pm 0.69$ & $4.24 \pm 0.71$ & $4.23 \pm 0.61$ & 4.762 & $\mathbf{0 . 0 0 9}$ \\
\hline
\end{tabular}

Tendo em conta principalmente a faixa etária mais nova, verifica-se que estes possuem uma orientação elevada para o ego, pela que a importância da comparação com a prestação dos companheiros, surge como uma das únicas formas de determinar e percepcionar os seus níveis de competência e sucessivamente, sucesso. O grupo dos 13-15 anos, demonstra ser aquele que possui níveis mais elevados de atitudes anti-desportivas e de orientação para o ego, conjuntamente com o grupo dos mais novos. A variável faixa etária, desta forma, assume-se como aquela com maior poder discriminante para o objecto de estudo.

O quadro 5 contém os resultados comparativos dos construtos objecto de estudo, quando confrontando os diferentes subgrupos da variável independente envolvimento desportivo.
Assim, na comparação entre praticantes e não praticantes desportivos, os resultados revelam no geral, que os indivíduos quando praticantes de uma modalidade colectiva ou individual, revelam maior vontade de praticar desporto e considerando sempre o respeito pelo adversário e regras, possuindo assim níveis mais elevados de orientação para a tarefa.

No que concerne a influência da orientação para a tarefa nas atitudes desportivas, a análise de regressão linear permitiu constatar que a orientação para a tarefa evidenciou-se como uma variável preditiva em mais de $16 \%$ de variância das atitudes desportivas $\left(r=0.404 ; r^{2}=0.163\right.$; $\alpha=0.404 ; \mathrm{t}=9.052 ; \mathrm{p}=0.000)$. Assim, assume-se como um preditor significativo e de considerável importância para a promoção do espírito desportivo em aulas de Educação Física. 


\section{Discussão}

A problemática que nos levou a realizar este estudo, há muito tempo que está emergente, e é estudada no contexto desportivo. Em vários países, têm-se analisado a importância dos valores no processo formativo dos jovens, enquanto futuros cidadãos de uma sociedade "desvirtuada" de comportamentos e princípios adequados. Como tal, incorporar princípios e valores de acordo com o fair-play numa sociedade cujo único valor é vencer independentemente dos meios usados, assume-se como uma "missão" muito difícil para os professores de Educação Física.

Os rapazes concordaram mais que as raparigas em comportamentos relacionados com condutas anti-desportivas e orientação para o ego, confirmando os resultados da literatura neste âmbito, que menciona que os rapazes aceitam com mais facilidade certos comportamentos ilegais, como sendo perfeitamente legítimos, face aos objectivos pretendidos na competição em causa ${ }^{10}$. Para compreender os comportamentos associados ao sexo em contexto desportivo, é fundamental ter em conta as diferenças culturais, bem como também, as expectativas da sociedade respeitante à sua participação e integração de certas normas e valores socioculturais ${ }^{15}$.

Quanto à análise das diferentes faixas etárias, estes valores não se assumem de forma linear, emergindo no entanto o facto da faixa etária com mais de 16 anos, ser aquela com níveis mais elevados de orientação para a tarefa e concordância com as atitudes desportivas. Por outro lado, os alunos dos 13 aos 15 anos assumem-se como sendo aqueles que mais concordam com atitudes anti-desportivas em aulas de Educação Física. Tal facto é concordante com Vallerand e Losier $^{14}$, que referem que os adolescentes estão mais predispostos a demonstrar uma atitude negativa perante a participação desportiva e mostram menor preocupação com o respeito pelas regras e oficiais de jogo. Concomitantemente, Duda et al. ${ }^{3}$ referiram que atletas orientados para o ego revelaram comportamentos contrários à demonstração de atitudes morais respeitantes ao espírito desportivo, pretendendo obter a vitória a "todo o custo".

Relativamente ao envolvimento desportivo, os alunos praticantes (quer seja modalidade colectiva ou individual), tendem a demonstrar maiores níveis de orientação para a tarefa, salientando que a prática desportiva contribuiu para o desenvolvimento integral dos jovens e como tal para a sua formação ético-moral. Tal é concordante com o estudo de Fernandes et al. ${ }^{4}$ realizado no contexto nacional, sugerindo-se uma correcta transmissão de valores através das situações criadas ao longo da vivência desportiva ${ }^{6}$. Desta forma, a competição tem de ser considerada como um instrumento pedagógico importante no processo de formação desportiva, extraindo desta as consequências indispensáveis à orientação da sua intervenção pedagógica. Competição significa treino periódico, esforço, atitude permanente de superação e perseguição permanente do melhor resultado. Logo, procurar a vitória não constitui qualquer problema, sendo inerente ao acto de competir. O problema está nos meios que se usam, assim como nas atitudes e comportamentos que se assumem quando aquele objectivo não é atingido ${ }^{12}$.

No que se refere à análise da influência preditiva, os resultados indicam que a orientação para a mestria, assume-se como um construto preditor significativo e importante para a promoção do espírito desportivo em aulas de Educação Física, visando a definição de atitudes e condutas morais, de forma a diminuir a incidência de casos de indisciplina neste contexto ${ }^{13}$.

Neste âmbito, é de fulcral importância a intervenção do professor de Educação Física/treinador na formação técnica e psicopedagógica dos jovens ${ }^{2}$. Para este processo de aprendizagem social entre os alunos e professores, Gonçalves ${ }^{8}$ refere diversos estudos desenvolvidos em Portugal e 
em países estrangeiros (Alemanha, Estados Unidos e Inglaterra), que apontam no sentido do professor/treinador constituir no desporto infanto-juvenil, o mais poderoso agente de socialização, em especial a partir dos 12-13 anos e de cuja actuação dependerá de forma relevante a qualidade das experiências que as crianças e jovens vivem no seu processo de formação desportiva. Assim, os professores/treinadores deverão considerar como primordiais, algumas áreas de intervenção como: (i) a persistente promoção dos valores éticos de conduta desportiva e princípios inerentes ao espírito desportivo; e, (ii) na defesa da ideia de que quem "usa a batota", a violência verbal ou física e que adopta processos que visam artificialmente aumentar o seu rendimento desportivo, para disso tirarem vantagem na competição, não compete de uma forma leal e correcta.

\section{Agradecimentos}

Este trabalho foi apresentado sob a forma de comunicação no $6 .^{\circ}$ Congresso Nacional de Educação Física. CNAPEF/SPEF, Lisboa

\section{Correspondência}

José Vasconcelos-Raposo

Rua Dr. Manuel Cardona

5000-558 Vila Real, Portugal

E-mail: j.vasconcelos.raposo@gmail.com

\section{Referências}

1. Bredemeier, B. e Shields, D. (1993). Moral psychology in the context of sport. In R. Singer, M. Murphey e K. Tennant (Eds.), Handbook of research on sport psychology (p. 587-599). New York: MacMillan.

2. Buñuel, P. S., Fuentes-Guerra, J. G. e Robles, A. S. (2000). Necesidad de formación del profesor/entrenador deportivo para conseguir conductas deportivas o de fair-play en la enseñanza del deporte. Askesis, 11 (3).

3. Duda, J., Olson, L. e Templin, T. (1991). The relationship of task and ego orientation to sportsmanship attitudes and the perceived legitimacy of injurious acts. Research Quarterly for Exercise and Sport, 62, 297-334.

4. Fernandes, H., Costa, H. e Moreira, M. (2003). Valores no desporto: Estudo exploratório em alunos da licenciatura em Educação Física e Desporto da UTAD. In A. Pereira (Ed.), Actas do Simpósio A actividade física do lazer ao rendimento: A estética, a saúde e o espectáculo. Lamego: ISPV.

5. Fonseca, A. M. e Biddle, S. (1995). Estudo inicial para a adaptação do TEOSQ à realidade portuguesa: Questionário sobre a orientação para a tarefa e para o ego no desporto (TEOSQp). In A.M. Fonseca (Ed.) (2001), A FCDEF-UP e a Psicologia do Desporto: Estudos sobre motivação. Porto: FCDEF-UP.

6. Gonçalves, C. (1991). A prática desportiva das crianças e dos jovens: Contributos para o seu alcance educativo e formativo. In J. Bento e A. Marques (Eds.), As ciências do desporto e a prática desportiva: Desporto na escola; Desporto na reeducaşão e reabilitação - Actas. Porto: FCDEF-UP.

7. Gonçalves, C. (1998). Relações entre características e crenças dos alunos e os seus comportamentos nas aulas de Educação Física. Tese de Doutoramento não publicada. Lisboa: UTL-FMH.

8. Gonçalves, C. (2000). Os treinadores e as aprendizagens sociais. Notícias do Espirito Desportivo (on-line), 8 (3), 1. < Disponível na Internet a 31/07/2005 em

http://www.cm-oeiras.pt> 
9. Hellison, D. (1987). Dreaming the possible dream: The rise and triumph of Physical Education. In J. Massengale (Ed.), Trends towards the future in Physical Education. Champaign: Human Kinetics.

10. Lemyre, P., Roberts, G. e Ommundsen, Y. (2002). Achievement goal orientations, perceived ability, and sportspersonship in youth soccer. Journal of Applied Sport Psychology, 14, 120-136.

11. Rosado, A. e Marques, R. (1999). Indisciplina em Educação Física: Estudo da variabilidade de comportamentos perturbadores em função do ano de escolaridade e do nível dos alunos em Educação Física. Ludens, 16 (2), 5-10.

12. Silva, J. (1983). The perceived legitimacy of rule violating behavior in sport. Journal of Sport Psychology, 5, 438-448.
13. Spray, C. e Wang, C. (2001). Goal orientations, self-determination and pupils' discipline in Physical Education. Journal of Sports Sciences, 19, 903-913.

14. Vallerand, R. e Losier, G. (1994). Self-determined motivation and sportsmanship orientations: An assessment of their temporal relationship. Journal of Sport \& Exercise Psychology, 16, 229-245.

15. Weinberg, R. e Gould, D. (1997). Psychologie du sport et de l'activité physique (p. 493-542). Quebéc: Editions Edisem/Vigot.

16. Weiss, M. R. (2000). Motivating kids in Physical Activity. President's Council on Physical Fitness and Sports Research Digest, 11 (3), 1-8. 